\title{
Outbreak of Resistant Acinetobacter baumannii - Measures and Proposal for Prevention and Control
}

\author{
Roberta Maia de Castro Romanelli ${ }^{1,2}$, Lenize Adriana de Jesus ${ }^{1}$, Wanessa Trindade Clemente ${ }^{1,2,3}$, Stella Sala Soares Lima ${ }^{1,2}$, Edna \\ Maria Rezende ${ }^{2,4}$, Rosane Luiza Coutinho ${ }^{1}$, Ricardo Luiz Fontes Moreira ${ }^{1}$, Francelli Aparecida Cordeiro Neves ${ }^{1}$ and Nelma de \\ Jesus Brás ${ }^{1}$ \\ ${ }^{1}$ Comissão de Controle de Infecção Hospitalar do Hospital das Clínicas da Universidade Federal de Minas Gerais (HC/UFMG); ${ }^{2}$ Grupo de \\ Estudo e Pesquisas em Infecção Associada ao Cuidado de Saúde e Epidemiologia Hospitalar GREPI.; ${ }^{3}$ Departamento de Propedêutica \\ Complementar da Faculdade de Medicina da Universidade Federal de Minas Gerais - UFMG; ${ }^{4}$ Escola de Enfermagem da Universidade \\ Federal de Minais; Belo Horizonte, MG, Brazil
}

\begin{abstract}
Acinetobacter baumannii colonization and infection, frequent in Intensive Care Unit (ICU) patients, is commonly associated with high morbimortality. Several outbreaks due to multidrug-resistant (MDR) A. baumanii have been reported but few of them in Brazil. This study aimed to identify risk factors associated with colonization and infection by MDR and carbapenem-resistant $A$. baumannii strains isolated from patients admitted to the adult ICU at HC/UFMG. A case-control study was performed from January 2007 to June 2008. Cases were defined as patients colonized or infected by MDR/carbapenem-resistant $A$. baumannii, and controls were patients without MDR/ carbapenem-resistant $A$. baumannii isolation, in a 1:2 proportion. For statistical analysis, due to changes in infection control guidelines, infection criteria and the notification process, this study was divided into two periods. During the first period analyzed, from January to December 2007, colonization or infection by MDR/carbapenem-resistant A. baumannii was associated with prior infection, invasive device utilization, prior carbapenem use and clinical severity. In the multivariate analysis, prior infection and mechanical ventilation proved to be statistically significant risk factors. Carbapenem use showed a tendency towards a statistical association. During the second study period, from January to June 2008, variables with a significant association with MDR/carbapenem-resistant A. baumannii colonization/infection were catheter utilization, carbapenem and third-generation cephalosporin use, hepatic transplantation, and clinical severity. In the multivariate analysis, only CVC use showed a statistical difference. Carbapenem and third-generation cephalosporin use displayed a tendency to be risk factors. Risk factors must be focused on infection control and prevention measures considering A. baumanni dissemination.
\end{abstract}

Key-Words: Acinetobacter baumannii, multidrug resistance, carbapenem, infection control, outbreak.

Acinetobacter baumannii is a ubiquitous aerobic Gramnegative bacterium with non-fermentative metabolism found in nature especially in humid locations, which is able to contaminate and persist on various surfaces, disseminating among patients and in diverse settings. It has been described as an important microorganism that frequently colonizes and infects patients in Intensive Care Units (ICU) worldwide. In this environment, A. baumannii can display multiple antimicrobial resistance profiles, which are responsible for significant morbimortality [1-3].

A. baumannii is an opportunistic pathogen, frequently involved in infection outbreaks, especially in the ICU. It is a common cause of sepsis, pneumonia, and urinary tract infection following hospitalization of seriously ill patients.

Usually, the strains are ssensitive to carbapenems, fluorquinolones, sulbactam associations, tigecycline, rifampicin, aminoglycosides, and polimixins. For empiric therapy, carbapenems are frequently the drug of choice, but ampicillin plus sulbactam, ciprofloxacin, aminoglycoside associations, rifampicin, or other drugs have also been used. Although resistance profile definitions vary in literature, it is

Received on 23 April 2009; revised 16 September 2009.

Address for correspondence: Dr.Roberta Maia de Castro Romanelli. Av. Alfredo Balena 110, Hospital das Clinicas - $1^{\circ}$ andar (CCIH). Zip code: 30 130-100. Phone: (31) 34099383 / FAX: (31) 25511500. E-mail: rmcromanelli@medicina.ufmg.br.

The Brazilian Journal of Infectious Diseases

2009;13(5):341-347. (C) 2009 by The Brazilian Journal of Infectious Diseases and Contexto Publishing. All rights reserved. considered that resistance to carbapenems is, in itself, sufficient to define an isolate as highly resistant [2,4-8].

According to the Center for Diseases Control and Prevention (CDC), an A. baumannii multidrug-resistant (MDR) strain is defined as resistant to one or more classes of antimicrobial agents, including carbapenems [9]. A minimal inhibitory concentration (MIC) of $=16 \mathrm{mg} / \mathrm{mL}$ for imipenem or meropenem is necessary to define carbapenem resistance [10]. The expression "MDR/carbapenem-resistant $A$. baumannii" was first used in 1991 during an outbreak at a hospital in New York City [11]. In 1998, in Taiwan, the term pandrug-resistant (PDR) A. baumannii was described, related to resistance to all antimicrobial agents available at that time, including carbapenems, all cephalosporins, aztreonam, aminoglycosides and ciprofloxacin [12].

MDR/carbapenem-resistant $A$. baumannii is associated with treatment challenges, emphasizing the importance of preventing and controlling the dissemination of this strain. Outbreaks due to MDR/carbapenem-resistant A. baumanii have been reported, mainly in ICUs, and treatment of these strains has been considered a global problem [13-15]. The Meropenem Yearly Susceptibility Test Information Collection (MYSTIC), which includes strains from several sites worldwide, describes A. baumannii sensitivity as $76.1 \%$ and $74.7 \%$ to meropenem and imipenem, respectively. Sensitivity is even lower for Latin American strains [16-17]. According to the Sentry Antimicrobial Surveillance Program, A. baumannii resistance was reported at rates of $14 \%, 68 \%, 65 \%$, and $48 \%$ to imipenem, ceftazidime, ciprofloxacin, and ampicillin plus 
sulbactam, respectively [18]. Although some studies describe increasing resistance to carbapenems and other drugs, achieving rates up to $90 \%$ [19-20].

In the resistance setting, some other drugs can be used for these infections and polimixin is the first choice [2-5]. Tigecycline, another treatment option, was only licensed by the Food and Drug Administration (FDA) for skin and abdominal infections. Further, there is no standard MIC to define A. baumanni sensitivity to tigecycline, and clinical resistance is also reported [2-5,21-25]. Recently, it was suggested that combination therapy could improve treatment outcome by a synergistic effect [26].

MDR/carbapenem-resistant $A$. baumannii has been identified in our hospital since 2004 and dissemination in different settings has been recognized. Considering the epidemiological importance of this microorganism, the Hospital Infection Control Committee (HICC) developed this study to determine the risk factors associated with colonization or infection.

\section{Material and Methods}

Study Design

This case-control study was performed in an adult ICU at the University Hospital from January 2007 to June 2008. Cases were defined as all patients colonized or infected by MDR/ carbapenem-resistant $A$. baumannii, as of the second day after ICU admission and until 48 hours after discharge from the Unit. Controls were patients admitted to the ICU during the study period from which MDR/carbapenem-resistant $A$. baumannii was not isolated, matched with a 2:1 proportion (controls:cases ratio).

Data were routinely collected by HICC, according to the Brazilian Ministry of Health [27] and National Healthcare Safety Network (NHSN) recommendations [28].

Microbiological Identification

Swabs and biological samples were cultured. All Acinetobacter strains identified in any biological material were cultivated by standard microbiological methods (Gram staining, colony and cell morphology, and biochemical tests). Susceptibility characterization was established by Clinical and Laboratory Standards Institute (CLSI) recommendations [10].

\section{Variables \\ Age, gender, device utilization (central venous catheter - CVC; mechanical ventilation - MV; indwelling urinary catheter - IUC), antimicrobial use (carbapenems, fluorquinolones, and third- generation cephalosporin), surgery in the 30 days prior to ICU admission, transplantation (heart, liver, or kidney), severity score at ICU admission (classified from A to $\mathrm{E}$ according to the Average Severity of Illness Score - ASIS), [29] and prior infection (regardless of microorganism or infection site) were studied.}

Statistical Analysis

Data were organized and analyzed using the Statistical Package for the Social Sciences (SPSS), version 13.0.
Proportion comparison was performed by Pearson's $\chi^{2}$ or Fisher's test and mean comparison with the t test. Statistical significance was reached when $p £ 0.05$, and in these variables the Odds Ratio (OR) was considered with a 95\% confidence interval (95\% CI). Univariate and multivariate analysis was performed, using length of stay in the ICU to match case and control groups in order to avoid a bias.

For statistical analysis, the study was divided into two periods (January - December 2007, and January - June 2008), since recommendations of infection control guidelines were altered by NHSN/CDC [28], with modifications in terms of infection criteria and notification process.

\section{Ethical Considerations}

This study was approved by the Institutional Research Ethical Committee. Privacy was guaranteed, and patients were identified by their hospital registration number. Only HICC personal involved in data collection and researchers had access to the information of all enrolled patients.

\section{Results}

Outbreak Description

From January 2004 to December 2006, in the ICU, the mean colonization/infection rate due to MDR/carbapenem-resistant A. baumannii was 0.5 case/month. After that, however, an increase in cases was identified and lead to a mean incidence of 5.3 cases/month in 2007 and 5.7 cases/month in 2008. This event was defined as an outbreak, since the mean incidence was above the control limit, defined as three standard deviations above the mean incidence (FIGURE 1).

First Period (January - December 2007)

- Fifty-one patients infected or colonized by MDR/ carbapenem-resistant $A$. baumannii were included and paired to 102 control patients, according to defined criteria.

- Univariate analysis: Colonization or infection by MDR/ carbapenem-resistant $A$. baumannii was associated with those patients who had prior infection $(\mathrm{p}<0.001)$, device utilization such as MV $(\mathrm{p}<0.001)$, CVC $(\mathrm{p}=0.001)$, and IUC $(\mathrm{p}=0.004)$, prior carbapenem use $(\mathrm{p}<0.001)$, and a severe ASIS score $(\mathrm{p}<0.001)$ (Table 1$)$.

- Multivariate analysis: prior infection $(\mathrm{p}=0.002)$ and MV use $(\mathrm{p}=0.003)$ continued to be significant risk factors. Carbapenem use showed a tendency towards a statistical association ( $\mathrm{p}=0.07)$ (Table 2).

Second Period (January to June 2008)

- Thirty-five cases were notified and 70 control patients were selected for analysis.

- Univariate analysis: variables with a significant association with MDR/carbapenem-resistant A. baumannii colonization or infection were CVC utilization ( $\mathrm{p}=0.02)$, carbapenem and third-generation cephalosporin use ( $\mathrm{p}=0.019$ and $\mathrm{p}=0.039$, respectively), liver transplantation $(\mathrm{p}=0.015)$, and severe ASIS score $(\mathrm{p}=0.041)$ (Table 3$)$. 
Table 1. Risk factor univariate analysis for MDR/carbapenem-resistant A. baumannii in the Intensive Care Unit, Hospital das Clínicas - UFMG, 2007.

\begin{tabular}{|c|c|c|c|c|c|}
\hline \multirow[t]{2}{*}{ Variable } & \multicolumn{2}{|c|}{ MDR/carbapenem-resistant $A$. baumannii } & \multirow[t]{2}{*}{$\mathbf{p}$} & \multirow[t]{2}{*}{ OR } & \multirow[t]{2}{*}{$95 \% \mathrm{CI}$} \\
\hline & $\begin{array}{c}\text { Case (51) } \\
\text { N (\%) }\end{array}$ & $\begin{array}{c}\text { Control (102) } \\
\text { N (\%) }\end{array}$ & & & \\
\hline \multicolumn{6}{|l|}{ Gender } \\
\hline M & $23(45.1)$ & $53(52)$ & 0.42 & 0.8 & $0.5-1.3$ \\
\hline $\mathrm{F}$ & $28(54.1)$ & $49(48)$ & & & \\
\hline \multicolumn{6}{|l|}{ Invasive devices } \\
\hline MV & $44(86.3)$ & $33(32.4)$ & $<0.001$ & 6.2 & $1.3-4.5$ \\
\hline CVC & $41(80.4)$ & $55(53.9)$ & 0.001 & 2.4 & $1.3-4.5$ \\
\hline IUC & $48(94.1)$ & $76(74.5)$ & 0.004 & 3.7 & $1.3-11.2$ \\
\hline \multicolumn{6}{|l|}{ ATB utilization } \\
\hline Fluorquinolones & $14(27.5)$ & $17(16.7)$ & 0.12 & 1.5 & $0.9-2.4$ \\
\hline $3^{\text {rd }}$ gen. cephalosporin & $9(17.7)$ & $14(13.7)$ & 0.52 & 1.2 & $0.7-2.1$ \\
\hline Carbapenem & $21(41.2)$ & $10(9.8)$ & $<0.001$ & 2.8 & $1.9-4.1$ \\
\hline \multicolumn{6}{|l|}{ Severity (ASIS) } \\
\hline A & $3(5.9)$ & $33(32.4)$ & $<0.001$ & - & - \\
\hline B & 0 & $10(9.8)$ & & & \\
\hline $\mathrm{C}$ & $18(35.3)$ & $34(33.3)$ & & & \\
\hline $\mathrm{D}$ & $23(45.1)$ & $21(20.6)$ & & & \\
\hline $\mathrm{E}$ & $7(13.7)$ & $4(3.9)$ & & & \\
\hline \multicolumn{6}{|l|}{ Transplantation } \\
\hline Heart & 0 & $4(3.9)$ & 0.15 & 1.5 & $1.4-1.7$ \\
\hline Liver & $1(2.0)$ & $4(3.9)$ & 0.67 & 0.6 & $0.1-3.5$ \\
\hline Kidney & $1(2.0)$ & $3(2.9)$ & 1.0 & 0.8 & $0.1-4.1$ \\
\hline Prior surgery & $34(66.7)$ & $63(61.8)$ & 0.55 & 1.2 & $0.6-2.5$ \\
\hline Prior infection & $36(70.6)$ & $26(25.5)$ & $<0.001$ & 3.5 & $2.1-5.9$ \\
\hline
\end{tabular}

Table 2. Risk factor multivariate analysis for MDR/carbapenem-resistant A. baumannii in the Intensive Care Unit, Hospital das Clínicas - UFMG, 2007.

\begin{tabular}{lccc}
\hline & $\mathbf{p}$ & $\mathbf{O R}$ & $\mathbf{9 5 \%}$ CI \\
\hline Prior infection & 0.002 & 4.190 & $1.69-10.41$ \\
MV & 0.003 & 6.045 & $1.86-19.69$ \\
CVC & 0.78 & .842 & $0.26-2.77$ \\
IUC & 0.88 & 1.133 & $0.22-5.85$ \\
Carbapenem & 0.07 & 2.660 & $0.92-7.66$ \\
Severity (ASIS) & & & - \\
A & 0.67 & - & \\
B & 0.99 & & \\
C & 0.99 & & \\
D & 0.99 & & \\
E & 0.99 & & \\
\hline
\end{tabular}

OR- Odds ratio; CI- confidence interval; MV- mechanical ventilation; CVC- central venous catheter; IUC- indwelling urinary catheter.

- Multivariate analysis: CVC use persisted with a statistical difference as a risk factor associated with MDR/ carbapenem-resistant $A$. baumannii colonization/infection $(\mathrm{p}=0.04)$. Carbapenem and third-generation cephalosporin use showed a tendency towards this association $(\mathrm{p}=0.07$ and $\mathrm{p}=0.09$, respectively) (Table 4 ).

\section{Discussion}

A. baumannii has emerged as an important nosocomial pathogen and outbreaks due to multiple-resistant strains have been difficult to control, especially in the ICU setting.

Although more consistent studies of Acinetobacter MDR risk factors are available in medical literature, only a few of 
Table 3. Risk factors for MDR/carbapenem-resistant A. baumannii in the Intensive Care Unit, Hospital das Clínicas - UFMG, 2008.

\begin{tabular}{|c|c|c|c|c|c|}
\hline \multirow[t]{2}{*}{ Variable } & \multicolumn{2}{|c|}{ MDR/carbapenem-resistant A. baumannii } & \multirow[t]{2}{*}{$\mathbf{p}$} & \multirow[t]{2}{*}{ OR } & \multirow[t]{2}{*}{$95 \% \mathrm{CI}$} \\
\hline & $\begin{array}{c}\text { Case (35) } \\
\mathrm{N}(\%) \\
\end{array}$ & $\begin{array}{c}\text { Control (70) } \\
\text { N (\%) } \\
\end{array}$ & & & \\
\hline \multicolumn{6}{|l|}{ Gender } \\
\hline M & 19(54.3) & $29(41.4)$ & 0.21 & 0.71 & $0.41-1.22$ \\
\hline $\mathrm{F}$ & $16(45.7)$ & $41(58.6)$ & & & \\
\hline \multicolumn{6}{|l|}{ Invasive devices } \\
\hline MV & $27(77.1)$ & $46(65.7)$ & 0.23 & 1.5 & $0.76-2.8$ \\
\hline CVC & $30(85.7)$ & $45(64.3)$ & 0.02 & 2.4 & $1.03-5.59$ \\
\hline IUC & $34(97.1)$ & $61(87.1)$ & 0.16 & 3.6 & $0.55-23.4$ \\
\hline \multicolumn{6}{|l|}{ ATB utilization } \\
\hline Fluorquinolones & $10(28.6)$ & $17(24.3)$ & 0.64 & 1.16 & $0.64-2.08$ \\
\hline $3^{\text {rd }}$ gen. cephalosporin & $13(37.1)$ & $13(18.6)$ & 0.015 & 1.79 & $1.06-3.03$ \\
\hline Carbapenem & $17(48.6)$ & $18(25.7)$ & 0.019 & 1.89 & $1.12-3.19$ \\
\hline \multicolumn{6}{|l|}{ Severity (ASIS) } \\
\hline A & $14(40)$ & $30(42.9)$ & 0.04 & - & - \\
\hline B & $1(2.9)$ & 0 & & & \\
\hline $\mathrm{C}$ & 0 & $11(15.7)$ & & & \\
\hline D & $6(17.1)$ & 13(18.6) & & & \\
\hline $\mathrm{E}$ & $14(40)$ & $16(22.9)$ & & & \\
\hline \multicolumn{6}{|l|}{ Transplantation } \\
\hline Heart & 1(2.86) & $7(10)$ & 0.26 & 0.36 & $0.56-2.28$ \\
\hline Liver & $10(28.6)$ & $7(10)$ & 0.015 & 2.07 & $1.23-3.48$ \\
\hline Kidney & $2(5.7)$ & $3(4.3)$ & 1.00 & 1.2 & $0.4-3.68$ \\
\hline Prior surgery & $19(54.3)$ & $37(52.9)$ & 0.89 & 1.04 & $0.60-1.80$ \\
\hline Prior infection & 18(51.4) & $27(38.6)$ & 0.21 & 1.4 & $0.82-2.42$ \\
\hline
\end{tabular}

MDR- multidrug resistance; N- number; OR- Odds ratio; CI- confidence interval; M- male; F- female; MV- mechanical ventilation; CVC- central venous catheter; IUC- indwelling urinary catheter; ATB- antibiotic; ASIS- Average Severity of Illness Score.

Table 4. Risk factor multivariate analysis for MDR/carbapenem-resistant A. baumannii in the Intensive Care Unit, Hospital das Clínicas - UFMG, 2008.

\begin{tabular}{lccc}
\hline & $\mathbf{p}$ & $\mathbf{O R}$ & $\mathbf{9 5 \%} \mathbf{C I}$ \\
\hline CVC & 0.04 & 3.61 & $1.03-12.57$ \\
Carbapenem & 0.07 & 2.57 & $0.92-7.18$ \\
3 $^{\text {rd }}$ gen. Cephalosporin & 0.09 & 2.38 & $0.85-6.64$ \\
Severity (ASIS) & & & - \\
$\quad$ A & 0.97 & - & \\
B & 0.99 & & \\
C & 0.99 & & \\
D & 0.99 & & \\
E & 0.99 & & \\
\hline
\end{tabular}

$\overline{\text { OR- Odds ratio; CI- confidence interval; CVC- central venous catheter; ASIS- Average }}$ Severity of Illness Score.

them have been published in Brazil, despite the increasing isolation of MDR Acinetobacter strains. The first study was published in 1999 by Levin et al., and focused on clinical approach [30]. Even 10 years later, there are still uncertainties as to adequate prevention, control, and treatment of resistant Acinetobacter strains in epidemic and endemic situations.

MDR A. baumanni strains have been regularly isolated in Latin American hospital institutions [31]. Recently, Pseudomonas and Acinetobacter have been presented in
Europe and the United States as responsible for nosocomial outbreaks, mainly in ICUs, with different infection sites and high resistance rates [32-34]. Since A. baumannii survives for prolonged periods under a wide range of environmental conditions, this microorganism is difficult to control [35].

It is possible that the acquisition of genetic elements, and among these, plasmids containing resistance genes such as metallo-beta-lactamase, could be related to the emergence of resistant strains. Although diverse resistance mechanisms as 
Figure 1. MDR/carbapenem-resistant A. baumannii cases of colonization/infection, in the Intensive Care Unit, Hospital das Clínicas - UFMG, 2007-2008.

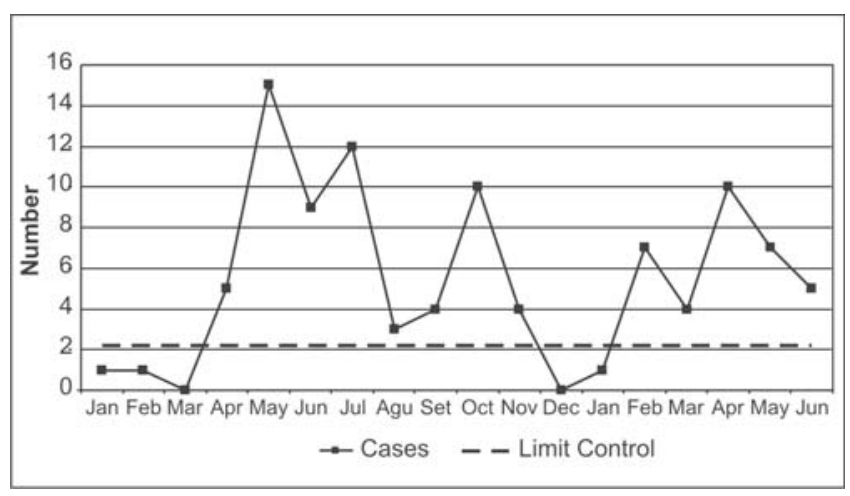

efflux, permeability alterations and genes are being studied, it is interesting to note that transferable genes, especially between Pseudomonas and Acinetobacter, can interfere by hindering the management of these microorganisms in healthcare settings [36]. In addition, in our setting, $P$. aeruginosa carbapenem-resistant strains were endemic during the period immediately prior to the study.

Colonization usually precedes infection. In order to avoid colonization, the prevention of microorganism dissemination is the target for infection control [37]. Controlling the risk factors associated with colonization and active surveillance measures (including swab cultures, contact precautions, cohorts, environmental control, patient decolonization, or antimicrobial prophylaxis and prescription control) are important actions for outbreak management $[3,4,38]$.

In the present study, to identify risk factors for MDR/ carbapenem-resistant Acinetobacter, 86 patients were compared to 172 non-colonized/infected patients. Colonization or infection was associated with prior infection and antibiotic use. During 2007, prior infection ( $\mathrm{p}=0.001)$ and carbapenem use $(p=0.001)$ and, in 2008, third- generation cephalosporin $(p=0.015)$ and carbapenem use $(p=0.019)$ were identified as risk factors. Antimicrobial treatments probably select resistant strains, as is shown by an association tendency in the multivariate analysis. Manikal et al., in a multicenter study, described an increase of MDR/carbapenem-resistant $A$. baumannii associated with the use of third-generation cephalosporins. These authors suggested that the use of one antimicrobial could improve resistance mechanisms to others [39]. Falagas and Kopterides observed that carbapenem and cephalosporin use were important risk factors for the selection of resistant strains [40]. Other studies have also shown, in univariate and multivariate analyses, that prior carbapenem use was associated with $A$. baumanni resistance [41,42].
Some articles have described an association between high carbapenem consumption rates, measured by the defined daily dose (DDD) and A. baumannii resistance. Carbapenems have long been regarded as the agents of choice, but resistance rates have risen [33]. Several studies have demonstrated that antimicrobial use can select resistant strains and elevated drug consumption is related to higher resistant rates. Marra et al. reported a carbapenem DDD (1000 patients/days) of 244.4 for a medical-surgical private hospital with an $88.5 \%$ A. baumannii resistance rate [43]. Another Brazilian study presented a 155.1 DDD index rate for carbapenem use, higher than expected [44]. At our institution, the carbapenem DDD index rate in the ICU was over the $90^{\text {th }}$ percentile, according to the National Nosocomial Infections Surveillance (NNIS) system [45].

In this series, the use of invasive devices was also associated with MDR/carbapenem-resistant $A$. baumannii. During 2007, MV, CVC, and IUC use displayed a statistical association ( $\mathrm{p}<0.001, \mathrm{p}=0.001$, and $\mathrm{p}=0.004$, respectively). In 2008, however, only CVC continued to be associated with the colonization/infection by MDR/carbapenem-resistant $A$. baumannii $(\mathrm{p}=0.022)$, even in a multivariate analysis $(\mathrm{p}=0.04)$. Several authors have correlated invasive devices and MDR/ carbapenem-resistant $A$. baumannii colonization, reinforcing the need for surveillance and control measures for these devices [13-15,40], mainly MV, as its habitat is a humid environment $[1,3,4,46]$. Moreover, patients who require invasive devices usually present with a more severe illness, demanding frequent medical interventions and have longer hospital stays, favoring colonization. As severity was analyzed, a statistical difference was also observed. Greater severity, according to the ASIS score, was identified in patients colonized/infected by MDR/carbapenem-resistant $A$. baumannii in the two periods of study $(\mathrm{p}<0.001$ and $\mathrm{p}=0.041$, respectively).

Although the outcome and mortality of Acinetobacter baumannii infections cannot be totally established, severity and multiple resistance could affect mortality rates. In the present study, 21 (30\%) of 70 patients colonized/infected by A. baumanni MDR/resistant to carbapenem died during the study period. Nevertheless, the presence of the microorganism was not a predictor for mortality. Although death showed no statistical association with the isolation of $A$. baumannii, the small number of cases precluded an adequate analysis. We point out that patients classified as Severity grade E by the ASIS score were not included.

However, several other studies have shown the association between mortality and colonization. In a case-control study that included 104 patients with MDR A. baumannii isolates and 104 controls, matched as per length of stay, an association with mortality was observed ( $\mathrm{p}=0.014)$, especially in the presence of infection $(\mathrm{p}<0.001)$ in a multivariate analysis. Colonization/infection by A. baumannii was a predictive factor of the need for $\mathrm{MV}$, and the use of this device was associated with mortality ( $\mathrm{p}=0.003$ ) [47]. An assessment of six case-control studies concluded that there is an association between 
infections by A. baumannii and mortality in hospitalized patients, regardless of the resistance profile, especially under inadequate antimicrobial therapy [48]. Another study carried out by Playford et al. described that patients with infections caused by carbapenem-resistant $A$. baumannii displayed a $20 \%$ higher rate of hospital mortality, with a 3.9 OR (95\% CI 1.4 to 10.7) when compared to those merely colonized [49]. Sunenshine et al., in a case-control study paired according to severity, described a higher mortality in patients infected by MDR A. baumannii when compared to controls $(\mathrm{p}<0.01)$ [50].

Infection Control measures, such as culture surveillance, contact precautions, cohorts, source identification, and environmental control, are effective in preventing microorganism dissemination [1,3,4,51,52]. These actions were recommended and monitored systematically by the HICC during the reported outbreak. An assistance team was directly informed on the outbreak data, microbiological results, and control recommendations. The participation and adherence of healthcare workers to preventive measures was extremely important in achieving control of the outbreak. However, infection control recommendations must be followed incessantly [53]. A booklet with all pertinent information was distributed to all professionals in order to sustain recommendations and to offer better patient assistance. In addition, the HICC antimicrobial audit and protocols for antimicrobial treatment definition, an A-I evidence level recommendation, are vital in order to avoid the emergence of microorganism resistance $[36,37,54]$. Another important suggestion is to avoid carbapenem use and indicate polimixin as an alternative treatment, whenever possible, during a MDR/ carbapenem-resistant $A$. baumannii outbreak [4].

The use of invasive devices (mainly MV and CVC), prior infections, use of antimicrobial agents (mostly carbapenem), and severity of clinical conditions can be associated with colonization or infection by MDR/carbapenem-resistant $A$. baumannii. These risk factors must be the focus of infection control actions, and the identification of colonized patients is extremely important to prevent microorganism dissemination.

\section{Acknowledgements}

The authors acknowledge the contributions of the ICU professionals, Hospital das Clínicas da Universidade Federal de Minas Gerais, and Kathleen Ruth Goldsmith Killing, MD, for reading and revising the text.

\section{References}

1. Fournier PE, Richet H. The Epidemiology and Control of Acinetobacter baumannii in Health Care Facilities. Clin Infect Dis 2006; 42:692-9.

2. Giamarellou H, Antoniadou A, Kanellakopoulou K. Acinetobacter baumannii: a universal threat to public health? Int J Antimicrob Agents 2008 Aug;32(2):106-19.

3. Munoz-Price LSs Weinstein RAa. Acinetobacter infection. N Engl J Medicine 2008 Mar 20;358(12):1271-81.

4. Urban C, Segal-Maurer S, Rahal J. Considerations in control and treatment of nosocomial infections due to multidrug-resistant Acinetobacter baumannii. Clin Infect Dis. 2003 May 15;36(10):1268-74.
5. Rice L. Challenges in identifying new antimicrobial agents effective for treating infections with Acinetobacter baumannii and Pseudomonas aeruginosa. Clin Infect Dis. 2006 Sep 1;43 Suppl 2:S100-5.

6. Song Jy, Kee Sy, Hwang Is, Seo Yb, Jeong Hw, Kim Wj, Cheong Hj. In vitro activities of carbapenem/sulbactam combination, colistin, colistin/rifampicin combination and tigecycline against carbapenem-resistant Acinetobacter baumannii. J Antimicrob Chemother 2007; 60: 317-322.

7. Tripodi Mf, Durante-Mangoni E, Fortunato R, Utili R, Zarrilli R. Comparative activities of colistin, rifampicin, imipenem and sulbactam/ampicillin alone or in combination against epidemic multidrug-resistant Acinetobacter baumannii isolates producing OXA-58 carbapenemases. Int J Antimicrob Agents 2007; 30: 537-540.

8. Bassetti M, Repetto E, Righi E, Boni S, Diverio M, Molinari Mp, Mussap M, Artioli S, Ansaldi F, Durando P, Orengo G, Bobbio Pallavicini F, Viscoli C. Colistin and rifampicin in the treatment of multidrug-resistant Acinetobacter baumannii infections. J Antimicrob Chemother 2008 Feb;61(2):417-20.

9. Siegel Jd, Rhinehart E, Jackson M, Chiarello. The Healthcare Infection Control Practices Advisory Committee Management of Multidrug - Resistant Organisms In Healthcare Settings Center for Diseases Control and Prevention (CDC), 2006. http:/ /www.cdc.gov/ncidod/dhqp/pdf/ar/mdroGuideline2006.pdf. Accessed em: 10/02/09.

10. Clinical Laboratory Standard Institute (CLSI). Performance Standards for Antimicrobial Suceptibility Testing; Seventeenth Information Suplement - M100s17. Data: Janeiro de 2007. Disponível em: www.clsi.org/source/orders/free/m100-s17.pdf Accessed: 29/04/2007.

11. Go Es, Urban C, Burns J, Kreiswirth B, Eisner W et al. Clinical and molecular epidemiology of Acinetobacter infections sensitive only to polymyxin B and sulbactam. Lancet 1994;344:132932.

12. Hsueh Pr, Teng Lj, Chen Cy, Chen Wh, Yu Cj, et al. Pandrugresistant Acinetobacter baumannii causing nosocomial infections in a university hospital, Taiwan. Emerg Infect Dis. 2002 Aug;8(8):827-32.

13. Wisplinghoff $\mathrm{H}$, Edmond Mb, Pfaller Ma, Jones Rn, Wenzel Rp, Seifert H. Nosocomial bloodstream infections caused by Acinetobacter species in United States hospitals: clinical features, molecular epidemiology, and antimicrobial susceptibility. Antimicrob Agents Chemother. 2000 Aug;44(8):2211-3.

14. Villegas Mv, Hartstein AI. Acinetobacter outbreaks 1977-2000. Infect Control Hosp Epidemiol. 2003; 24:284-95.

15. Garnacho-Montero J, Ortiz-Leyba C, Fernandez, E. Acinetobacter baumannii ventilator-associated pneumonia: epidemiological and clinical findings. Intensive Care Med, 2005 May; 31 (5): 649-55.

16. Rhomberg Pr, Jones RN. Contemporary activity of meropenem and comparator broad-spectrum agents: MYSTIC program report from the United States component (2005). Diagn Microbiol Infect Dis. 2007; 57 (2): 207-215.

17. Unal S, Garcia-Rodriguez J. Activity of meropenem and comparators against Pseudomonas aeruginosa and Acinetobacter spp. isolated in the MYSTIC Program, 2002-2004. Diagn Microbiol Infect Dis. 2005; Volume 53, Issue 4, 265 - 271.

18. Gales Ac, Jones Rn, Sader HS. Global assessment of the antimicrobial activity of polymyxin B against 54731 clinical isolates of Gram-negative bacilli: report from the SENTRY antimicrobial surveillance programme (2001-2004). Clin Microbiol Infect 2006; 12 (4): 315 - 321.

19. Souli M, Flora Vk, Koratzanis E, Antoniadou A, Giannitsioti E, Evangelopoulou P, Kannavaki S, Giamarellou H. In vitro Activity of Tigecycline against Multiple-Drug-Resistant, Including PanResistant, Gram-Negative and Gram-Positive Clinical Isolates from Greek Hospitals. Antimicrob Agents Chemother, September 2006; 50 (9): 3166-3169. 
20. Falagas Me, Mourtzoukou Eg, Polemis M, The Greek System For Surveillance Of Antimicrobial Resistance* and Vatopoulos AC. Trends in antimicrobial resistance of Acinetobacter baumannii clinical isolates from hospitalised patients in Greece and treatment implications. Clin Microbiol Infect 2007; 13 (8): $816-819$.

21. Frampton Je, Curran MP. Tigecycline. Drugs 2005; 65 (18): 2623 2635.

22. Livermore Dm. Tigecycline: what is it, and where should it be used? J Antimicrob Chemother. 2005 Oct;56(4):611-4.

23. Halstead Dc, Abid J, Dowzicky Mj. Antimicrobial susceptibility among Acinetobacter calcoaceticus-baumannii complex and Enterobacteriaceae collected as part of the Tigecycline Evaluation and Surveillance Trial. J Infect. 2007 Jul;55(1):4957.

24. Jones Rn, Ferraro Mj, Reller Lb, Schreckenberger Pc, Swenson Jm, Sader Hs. Multicenter studies of tigecycline disk diffusion susceptibility results for Acinetobacter spp. J Clin Microbiol. 2007 Jan;45(1):227-30.

25. Navon-Venezia S, Leavitt A, Carmeli Y. High tigecycline resistance in multidrug-resistant Acinetobacter baumannii. J Antimicrob Chemother. 2007 Apr;59(4):772-4.

26. Petrosillo N, Ioannidou E, Falagas ME. Colistin monotherapy $v s$. combination therapy: evidence from microbiological, animal and clinical studies. Clin Microbiol Infect 2008; 14: 816-827.

27. Brasil. Ministério Da Saúde. Portaria MS 2.616 / 98, que regulamenta as ações de controle de infecção hospitalar no país, em substituição a Portaria MS 930 / 92. Brasília, 12 de maio de 1998.

28. Center For Diseases Control And Prevention (CDC). The National Healthcare Safety Network (NHSN) Manual. Patient Safety Component Protocol. From: January 2008. Available at: http:/ /www.cdc.gov/ncidod/dhqp/pdf/nhsn/NHSN_Manual_ PatientSafetyProtocol_CURRENT.pdf. Accessed: maio de 2008.

29. Emori Tg, Culver Dh, Horan TC, et al. National Nosocomial Infections Surveillance System (NNIS): description of surveillance methods. Am J Infect Control 1991;19:19-35.

30. Levin As, Barone Aa, Penço J, Santos Mv, Marinho Is, Arruda Ea, Manrique Ei, Costa SF. Intravenous colistin as therapy for nosocomial infections caused by multidrug-resistant Pseudomonas aeruginosa and Acinetobacter baumannii. Clin Infect Dis 1999 May; 28(5):1008-11.

31. Sader Hs, Castanheira M, Mendes Re, Toleman M, Walsh Tr, Jones Rn. Dissemination and diversity of metallo-betalactamases in Latin America: report from the SENTRY Antimicrobial Surveillance Program. Int J Antimicrob Agents 2005 Jan; 25(1):57-61.

32. Souli M, Galani I, H Giamarellou H. Emergence of extensively drug-resistant and pandrug-resistant Gram-negative bacilli in Europe. Eurosurveillance, 2008 Nov; 13(47): 1-11.

33. Karageorgopoulos De, Falagas ME. Current control and treatment of multidrug-resistant Acinetobacter baumannii infections. Lancet Infect Dis 2008 Dec; 8(12):751-62.

34. Lockhart Sr, Abramson Ma, Beekmann Se, Gallagher G,Riedel S, Diekema Dj, Quinn Jp, Doern GV. Antimicrobial Resistance among Gram-Negative Bacilli Causing Infections in Intensive Care Unit Patients in the United States between 1993 and 2004. J Clin Microbiol, 2007 Oct 45 (10): 3352-3359.

35. Maragakis Ll, Perl TM. Acinetobacter baumannii: Epidemiology, Antimicrobial Resistance, and Treatment Options. Clin Infect Dis 2008 Apr; 46(8):1254-63.

36. Lee Mf, Peng Cf, Hsu Hj, Chen Yh. Molecular characterisation of the metallo-beta-lactamase genes in imipenem-resistant Gramnegative bacteria from a university hospital in southern Taiwan. Int J Antimicrob Agents 2008 Dec;32(6):475-80.

37. Kollef Mh, Fraser Vj. Antibiotic resistance in the intensive care unit. Ann Intern Med. 2001 Feb 20;134(4):298-314.
38. Dellit Th, Owens Rc, Mcgowan Je et al. Infectious Diseases Society of America and the Society for Healthcare Epidemiology of AmericaGuidelines for Developing an Institutional Program to Enhance Antimicrobial Stewardship. Clin Infect Dis 2007; 44:159-77.

39. Manikal Vm, Landman D, Saurina G et al Endemic carbapenemresistant Acinetobacter species in Brooklyn, New York: citywide prevalence, interinstitutional spread, and relation to antibiotic usage. Clin Infect Dis. 2000 Jul;31(1):101-6.

40. Falagas Me, Kopterides P. Risk factors for the isolation of multidrug-resistant Acinetobacter baumannii and Pseudomonas aeruginosa: a systematic review of the literature. J Hospital Infection, 2006; 64: 7-15.

41. Playford Eg, Craig Jc, Iredell JR. Carbapenem-resistant Acinetobacter baumannii. in intensive care unit patients: risk factors for acquisition, infection and their consequences. Journal of Hospital Infection 2007 65, 204-211.

42. Baran G, Erbay A, Bodur H, Onguru P, Akinci E, Balaban N, Cevik MA. Risk factors for nosocomial imipenem-resistant Acinetobacter baumannii infections. International Journal of Infectious Diseases 2008; 12, 16-21.

43. Marra Ar, De Almeida Sm, Correa L, Silva M Jr, Martino Md, Silva $\mathrm{Cv}$, Rodrigues Cal Rg, Edmond Mb, Dos Santos Of. The effect of limiting antimicrobial therapy duration on antimicrobial resistance in the critical care setting. Am J Infect Control 2008 Nov 3.

44. Santos Ef, Laura-Pires L, Pereira Mg, Silva Ae, Rodrigues Ip, Maia MO. Use of antibacterial agents in an intensive care unit in a hospital in Brazil Braz J Infect Dis 2007 Jun; 11(3): 355-9.

45. Center For Diseases Control And Prevention (CDC). Division of Healthcare Quality Promotion, National Center for Infectious Diseases. National Nosocomial Infections Surveillance (NNIS) System Report, data summary from January 1992 through June 2004, issued October 2004. Am J Infect Control 2004; 32(8):470-85.

46. Dellit Th, Chan Jd, Skerrett Sj, Nathens AB. Development of a Guideline for the Management of Ventilator-Associated Pneumonia Based on Local Microbiologic Findings and Impact of the Guideline on Antimicrobial Use Practices. Infection Control and Hospital Epidemiology 2008; vol. 29 (6): 525-533.

47. Abbo A, Carmeli Y, Navon-Venezia S, Siegman-Igra I, Schwaber MJ. Impact of multi-drug-resistant Acinetobacter baumannii on clinical outcomes. Eur J Clin Microbiol Infect Dis 2007; 26:793800.

48. Falagas M, Rafailidis PI. Attributable mortality of Acinetobacter baumannii: no longer a controversial issue. Critical Care 2007, 11 (3): 134

49. Playford Eg, Craig Jc, Iredell JR. Carbapenem-resistant Acinetobacter baumannii. in intensive care unit patients: risk factors for acquisition, infection and their consequences. Journal of Hospital Infection 2007 65, 204-211.

50. Sunenshine Ra, Wright Mo, Maragakis LL. Multidrug-resistant Acinetobacter infection mortality rate and length of hospitalization. Emerg Infect Dis 2007; 13:97-103.

51. Feely Tw, Du Moulin Gc, Hedley J, et al. Aerosol polymyxin and pneumonia in seriously ill patients. N Engl J Med 1975; 93:4715.

52. Maddison J, Dodd M, Webb AK. Nebulized colistin causes chest tightness in adults with cystic fibrosis. Respir Med 1994; 88:1457.

53. Siegel Jd, Rhinehart E, Jackson M, Chiarello L, And The Healthcare Infection Control Practices Advisory Committee, 2007. Center of Diseases Control and Prevention (CDC). Guideline for Isolation Precautions: Preventing Transmission of Infectious Agents in Healthcare Settings, June 2007. Disponível em: http://www.cdc.gov/ ncidod/dhqp/pdf/isolation2007.pdf. Acessado em 15/07/2007.

54. Kollef MH. Optimizing antibiotic therapy in the intensive care unit setting. Critical Care August 2001; 5 (4): 189-195. 